\title{
Estudio químico-cuán-
}

tico de los defectos

producidos por las

vacancias de oxígeno

\section{en los cristales de}

\section{Titanato de plomo}
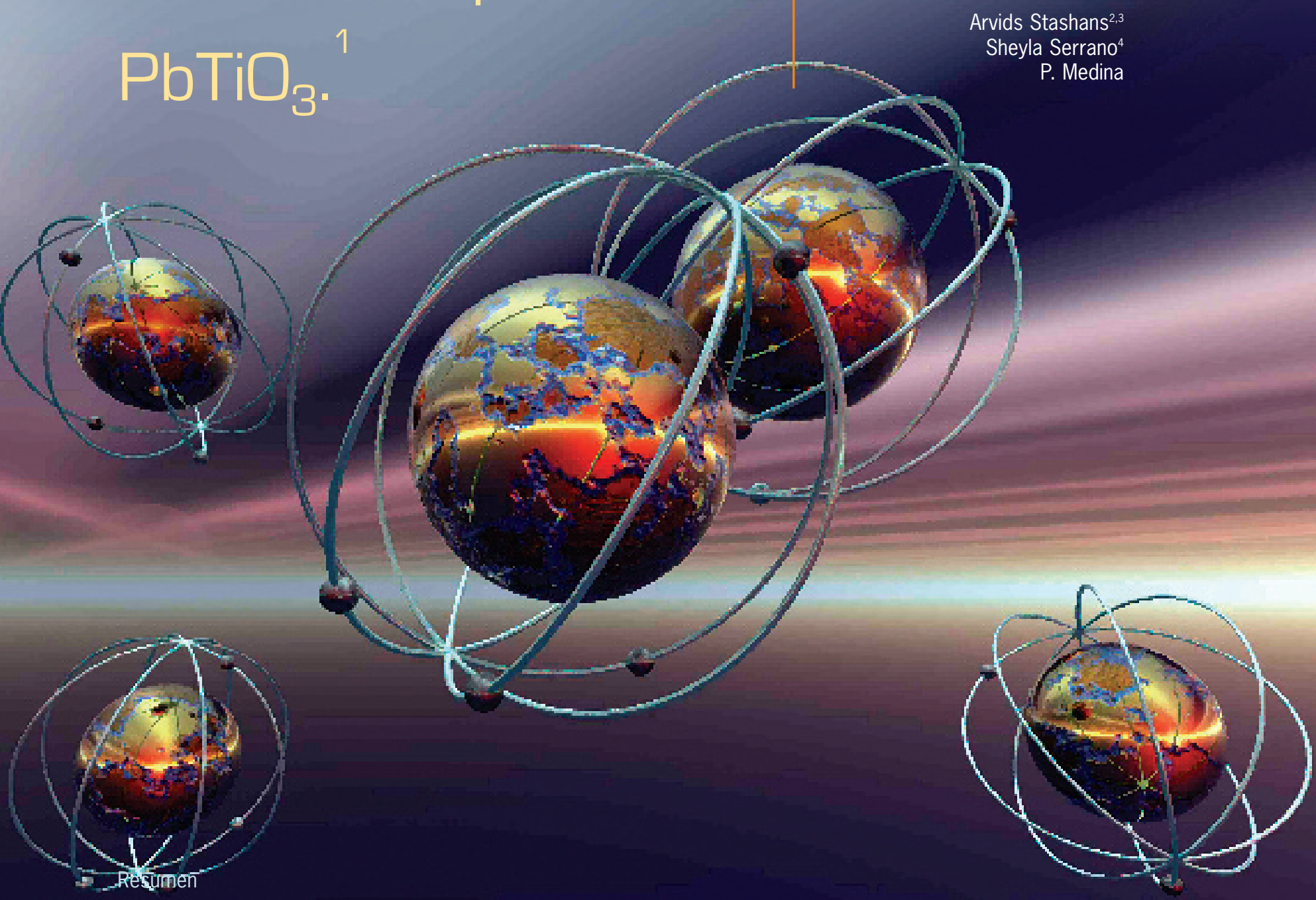

Se realiza una investigación teórica mediante simulaciones químico-cuánticas en la estructura cristalina del semiconductor tipo perovskita Titanato de Plomo PbTiO3 en su fase cúbica, generando vacancias de átomos de oxígeno y $\mathrm{F}$-centros. Los desplazamientos de los átomos vecinos, el incremento de las propiedades ópticas y eléctricas, las energías producto de la relajación de la red cristalina y otros nuevos efectos debido a la presencia de estos defectos se detallan y analizan. Además se presenta una comparación con estudios similares e importantes conclusiones de los resultados.

Palabras clave: Estructura electrónica, $\mathrm{PbTi03,} \mathrm{defectos} \mathrm{puntuales,} \mathrm{F-centros.}$ 


\section{INTRODUCCIÓN}

Desde el descubrimiento de la ferroelectricidad en la década de los cuarentas, la investigación científica de los cristales titanatos del tipo perovskita ha aumentado inmensamente. Debido a sus importantes propiedades, los titanatos incitan nuevas investigaciones científicas y el desarrollo de aplicaciones de alta tecnología alternativas en muchas ramas de la ingeniería. En particular, los cristales de $\mathrm{PbTiO}_{3}$ se caracterizan por poseer un campo de polarización reversible no volátil, que ha sido exitosamente utilizado en dispositivos de almacenamiento de memoria de radiación(1). Las propiedades piezoeléctricas, dieléctricas y ferroeléctricas del titanatos de plomo lo hacen atractivo en la tecnología debido a un gran número de otras aplicaciones como son los dispositivos de ondas acústicas de superficie, mecanismos micro-mecánicos y aparatos de campo ferroeléctrico, así como dispositivos de sonar y ultrasónicos.

Por otro lado, es bien conocido que los defectos puntuales como las vacancias y los F-centros juegan un rol muy importante dentro las aplicaciones de estos avanzados materiales. En particular, en la creación experimental de titanatos tipo perovskita, se conoce que las vacancias de oxígeno no pueden ser evitadas y que los F-centros son muy comunes. Estos defectos puntuales tienen una dramática influencia en las propiedades piezoeléctricas y ferroeléctricas de este importante material. $Y$, sin embargo, éstas no han sido estudiadas lo suficiente. Así, nuestro propósito es el estudio de los cristales de $\mathrm{PbTiO} 3$ que contienen estos defectos.

La estructura de un titanato de plomo perfecto es muy simple, tiene una simetría cúbica, con una red de octaedros de oxígeno interpenetrados con una red cúbica de cationes de Plomo $(\mathrm{Pb})$ y titanio (Ti). Los titanios se ubican en el centro de cada uno de los octaedros de Oxígeno (O), mientras que los Plomos se ubican en cada uno de los cuatro lados de los octaedros. A una temperatura inferior a los 766K, los cristales de $\mathrm{PbTiO}_{3}$ cambian a una fase tetragonal y se convierten en un material ferroeléctrico, así que las dos fases de este material deben ser investigadas. En este trabajo, se estudia este cristal utilizando el método de Hartree-

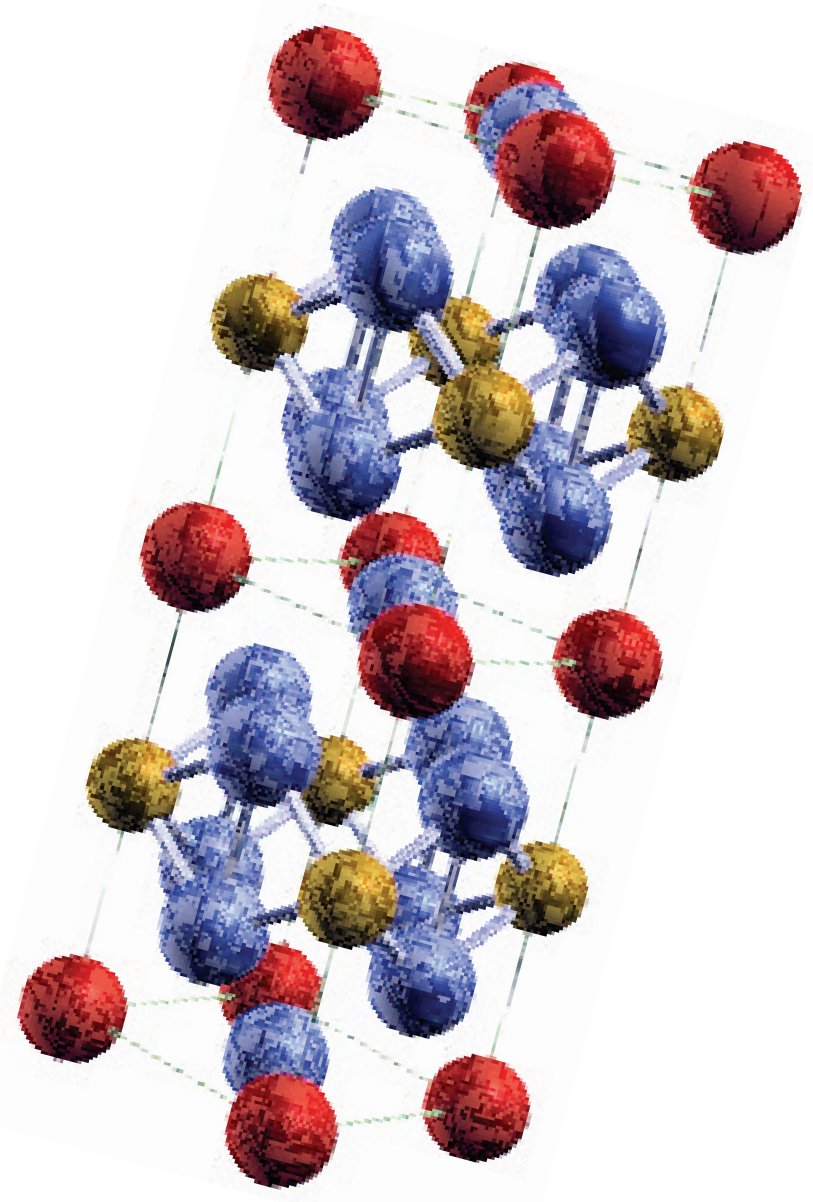

1 Resumen y traducción de la investigación realizada por los autores y publicada en la revista internacional Physica B, del editorial Elsevier de Inglaterra www.elsevier.com/locate/physb, recibida en octubre del 2005 y aceptada en diciembre del mismo año. Se encuentra disponible en Internet gracias a Science Direct www.sciencedirect.com, bajo el código Physica B 381 (2006) 82-89

2 Centro de Investigación de Física de la Materia Condensada, Corporación de Física Fundamental y Aplicada, Apartado 17-12-637.

3 Laboratorio de Física, Escuela de Electrónica y Telecomunicaciones, Universidad Técnica particular de Loja, Apartado 11-01-608 Loja, Ecuador.

4 Escuela de Ingenierías, Universidad Politécnica Salesiana, Campus Sur, Apartado 17-12-536 Quito, Ecuador. 
Fock, desarrollado para estudios sobre cristales y el modelo de celda unitaria grade periódica, large unit cell (LUC). Es importante notar que en nuestro método, el patrón de la distribución de la densidad electrónica en la vecindad del defecto esta dado dentro de un campo auto consistente self-consistent-field (SCF). Que nos da una información muy confiable acerca de la influencia de éste defecto en la la banda electrónica del cristal estudiado. Este método ya ha sido aplicado exitosamente para estudiar estos defectos en diferentes óxidos. Algunos ejemplos incluyen cálculos en estados base y estados excitados de $\mathrm{F}$ centros en cristales de corundum $\left(\mathrm{Al}_{2} \mathrm{O}_{3}\right)$ y defectos por vacancias de oxígeno en cristales de $\mathrm{BaTiO}_{3}$, y las superficies $\left(\begin{array}{lll}0 & 0 & 1\end{array}\right)$ (2) y muchos otros.

\section{BREVE RESUMEN DE NUESTRO MÉTODO}

Esta investigación explora un método químico-cuántico creado para estructuras periódicas basado en la teoría del Orbital Molecular (MO). Utilizando el código computacional SYM-SYM, se calcula la banda electrónica y la energía total del cristal perfecto gracias a MO y con la combinación lineal de los orbitales atómicos (AOs). Este método ha sido muy útil en el tratamiento de estructuras electrónicas y espaciales de un gran número de cristales, alrededor de 35, desde materiales iónicos simples hasta las complicadas estructuras con enlaces químicos mezcla de iónicos y covalentes. Además se puede predecir de la geometría del cristal con impurezas dopantes. Este hecho ha permitido resolver verdaderas incógnitas científicas. Asimismo es valioso mencionar que gracias a la fiel reproducción de la geometría del cristal, problemas fundamentales en el estudio de cristales como el Óxido de Titanio $\mathrm{TiO}_{2}$ fueron exitosamente resueltos.

Así, se realizó una parametrización de la red en la fase cúbica del cristal(3), estos parámetros fueron optimizados utilizando la estructura de la banda electrónica: ancho de la banda prohibida gap, anchos de las bandas de valencia, densidad de estados (DOS) de las bandas energéticas y geometría de la red cristalina. Nuestros resultados teóricos lograron exacta concordancia con los obtenidos experimentalmente en otros estudios. Inclusive

\begin{tabular}{|cccccc|}
\hline \multicolumn{7}{c}{ Atom } & $\mathrm{AO}$ & $\zeta$ & $E_{\text {neg }}$ & $-\beta$ & $p^{\circ}$ \\
\hline $\mathrm{Pb}$ & $6 \mathrm{~s}$ & 1,9 & 23,82 & 0,3 & 1,8 \\
& $6 \mathrm{p}$ & 1,85 & 16,8 & 0,3 & 0,2 \\
& $5 \mathrm{~d}$ & 2,90 & 36,8 & 29,0 & 2,00 \\
& $4 \mathrm{~s}$ & 1,75 & 1,5 & 0,8 & 0,55 \\
$\mathrm{Ti}$ & $4 \mathrm{p}$ & 1,70 & $-2,0$ & 0,8 & 0,22 \\
& $3 \mathrm{~d}$ & 1,88 & 0,07 & 8,0 & 0,55 \\
& $2 \mathrm{~s}$ & 2,27 & 4,5 & 16,0 & 1,974 \\
& $2 \mathrm{p}$ & 1,86 & $-12,6$ & 16,0 & 1,96 \\
\hline
\end{tabular}

Tabla 1.

Parámetros numéricos hallados en el presente artículo $\xi(a . u)$, $E_{\text {neg }}(\mathrm{eV}), \beta(\mathrm{eV}), \mathrm{p}^{\circ}(\mathrm{e})$ 


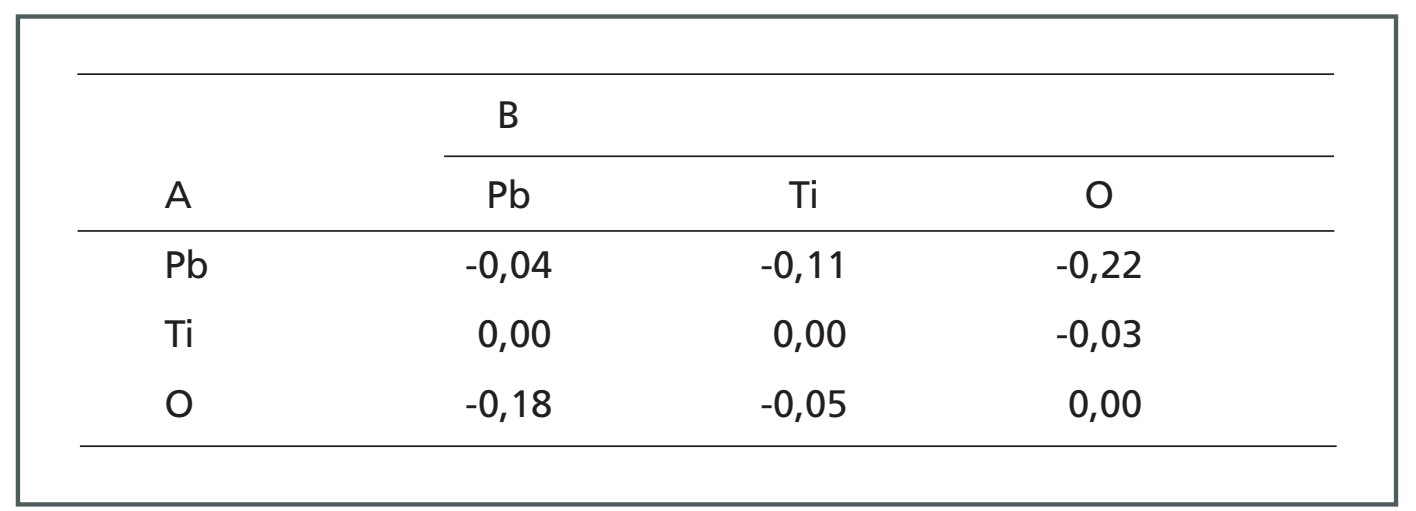

Tabla 2.

Parámetros de dos centros hallados en el presente estudio $\alpha_{\mu \beta}\left(a . u^{-1}\right)$

reproducimos la hibridización entre el orbital $3 p$ del titanio y el $2 p$ del oxígeno en la fase tetragonal, cubriendo así satisfactoriamente nuestras expectativas. Los parámetros obtenidos se muestran en las Tablas 1 y 2.

\section{RESULTADOS Y DISCUSIÓN:}

Estudiaremos las distorsiones de la red cristalina debido a las vacancias de oxígeno y F-centros: la estructura de la banda electrónica y las energías de absorción, para el estudio utilizamos una supercelda de 40 átomos que corresponde a una extensión de ocho veces la celda primitiva $(2 \times 2 \times 2)$.
Una vacancia de oxígeno es una imperfección de la red donde se saca a un ión de oxígeno. Los átomos vecinos a la vacancia se ajustan a nuevas posiciones de equilibrio, debido a la ausencia de carga negativa, la región de la vacancia se convierte entonces en una región de carga positiva. Es decir que la causa de los desplazamientos es la fuerza electrostática de Coulomb.

Estos desplazamientos son simétricos, debido a la simetría cúbica del cristal. Los ocho átomos de oxígenos adyacentes se acercan simétricamente al defecto en $0,14 \AA$ a lo largo de las direcciones $<11$ $0>$, como se muestra en la Figura 1.

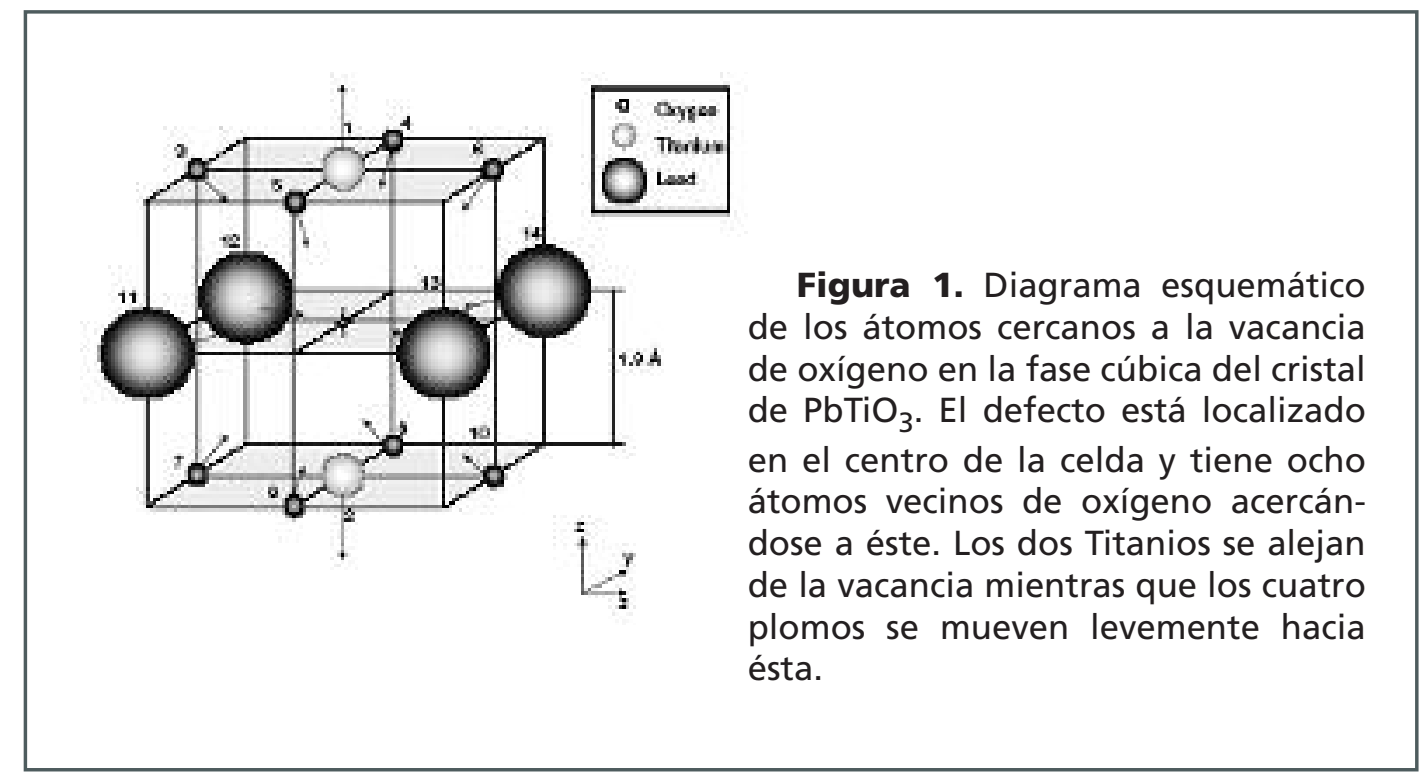


Claramente, debido a la fuerza electrostática de Coulomb, logrando así que los átomos de oxígeno se vuelvan menos negativos (Ver Tabla 3).

Esto implica que sus envolturas electrónicas se expanden hacia la región de la vacancia. Los dos átomos de titanio se alejan del defecto $0,17 \AA$ obedeciendo la misma ley. Esta relajación es la mayor de todos los átomos, además de un notable cambio de carga. En el caso de los cuatro átomos de Plomo, encontramos movimientos casi imperceptibles hacia la vacancia en $0,07 \AA$ debido a su tamaño, estos átomos aparentemente llenan el espacio vacío, acercándose hacia la vacancia. Al calcular las energías de relajación antes y después de la vacancia se encontró un valor igual 11,5 eV.

Para simular los F-centros, se colocan dos electrones en el lugar de la vacancia, sin asumir si se localiza o se de-localizan. Debido a la presencia negativa de los electrones, la carga total del sistema es cero, la principal causa de las relajaciones electrónicas es la relocalización de la densidad electrónica. Como se puede apreciar en la Tabla 3, se nota que los electrones se localizan en la región de la vacancia, muy cercanos a los átomos de Titanio. Tal como ocurrió antes, los movimientos producidos son simétricos (Ver Figura 2) los ocho oxígenos se mueven $0,05 \AA$ hacia el F-centro afirmando la teoría de que es la fuerza de Coulomb la responsable de los movimientos. Los titanios se alejan del defecto en $0,17 \AA$ y los plomos se alejan también en $0,05 \AA$. Estos hechos nos hacen afirmar que los dos electrones se localizan en la región de la vacancia causando un solapamiento entre ellos, y los electrones vecinos de los átomos de plomo, generando sus desplazamientos. La energía de relajación en este caso fue de 9,81 eV, 1,69 eV menos que en el caso de la vacancia, lo cual explica menos relajaciones en la red cristalina.

La variación de la energía de absorción, $\triangle \mathrm{SCF}$ entre los estados fundamentales y excitados del F-centro es de 2,3eV.

\begin{tabular}{|c|c|c|c|c|c|c|}
\hline \multirow[t]{3}{*}{ Atom } & \multirow{2}{*}{\multicolumn{2}{|c|}{$\begin{array}{l}\text { Oxygen vacancy } \\
\text { Charge }\end{array}$}} & \multirow{3}{*}{ Displacement } & \multicolumn{3}{|l|}{ F-center } \\
\hline & & & & \multicolumn{2}{|l|}{ Charge } & \multirow[t]{2}{*}{ Displacement } \\
\hline & Perfect crystal & vacancy & & Perfect crystal & F-center & \\
\hline $\mathrm{Ti}(1)$ & 2,48 & 2,38 & 0,17 & 2,48 & 1,87 & 0,17 \\
\hline $\mathrm{O}(4)$ & $-1,39$ & $-1,38$ & 0,14 & $-1,39$ & $-1,39$ & 0,05 \\
\hline $\mathrm{O}(5)$ & $-1,39$ & $-1,38$ & 0,14 & $-1,39$ & $-1,39$ & 0,05 \\
\hline $\mathrm{O}(6)$ & $-1,39$ & $-1,38$ & 0,14 & $-1,39$ & $-1,38$ & 0,05 \\
\hline $\mathrm{O}(7)$ & $-1,39$ & $-1,38$ & 0,14 & $-1,39$ & $-1,38$ & 0,05 \\
\hline $\mathrm{O}(8)$ & $-1,39$ & $-1,38$ & 0,14 & $-1,39$ & $-1,39$ & 0,05 \\
\hline$O(9)$ & $-1,39$ & $-1,38$ & 0,14 & $-1,39$ & $-1,39$ & 0,05 \\
\hline $\mathrm{Pb}(13)$ & 1,70 & 1,71 & 0,07 & 1,70 & 1,66 & 0,05 \\
\hline $\mathrm{Pb}(14)$ & 1,70 & 1,71 & 0,07 & 1,70 & 1,66 & 0,05 \\
\hline
\end{tabular}

Tabla 3.

Cargas en los átomos (en e) en la fase cúbica del $\mathrm{PbTiO}_{3}$, tanto en la red perfecta como en la red con la vacancia de oxígeno y con del F-centro, así como los desplazamientos de los átomos vecinos (en $\AA$ ) 


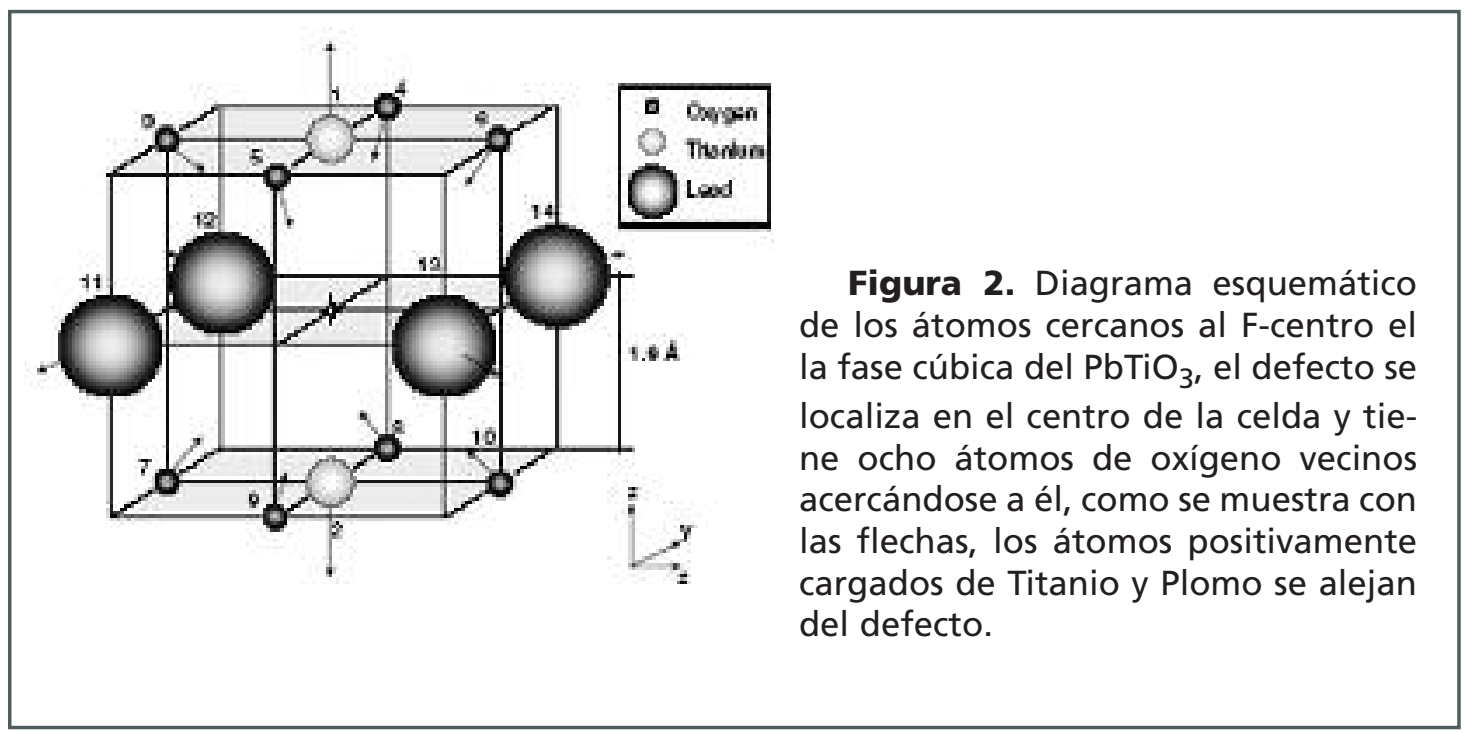

\section{CONCLUSIONES}

Este artículo presenta un estudio de las vacancias de oxígeno y los $\mathrm{F}$ centros en uno de los materiales más importantes en la tecnología, el $\mathrm{PbTiO}_{3}$, en su fase cúbica, utilizando un método químico-cuántico desarrollado para cristales y probado antes en varios cristales. Al estudiar las propiedades ópticas, electrónicas y estructurales se nota el rol predominante de la Fuerza de Coulomb en los movimientos e interacciones de los átomos, especialmente en el caso de las vacancias, para los F-centros, se considera además la importancia del enlace químico en los planos atómicos, especialmente en el caso del Titanio y del oxígeno.

\section{REFERENCIAS}

1. J.F. Scout, C.A Paz de Araujo, Science 246 (1989) 1400

2. C.Duque, A. Stashans, Physica B 336 (2003) 227

Stashans, Sheyla Serrano, Surf. Sci. 479 (2002) 285

Stashans, Sheyla Serrano, J. Ortiz, SPIE Proc. 5122 (2003) 264

3. A Stashans, C. Zambrano, A Sánchez, LM. Prócer, Ing. J. Quantum Chem. 87 (2002) 145.

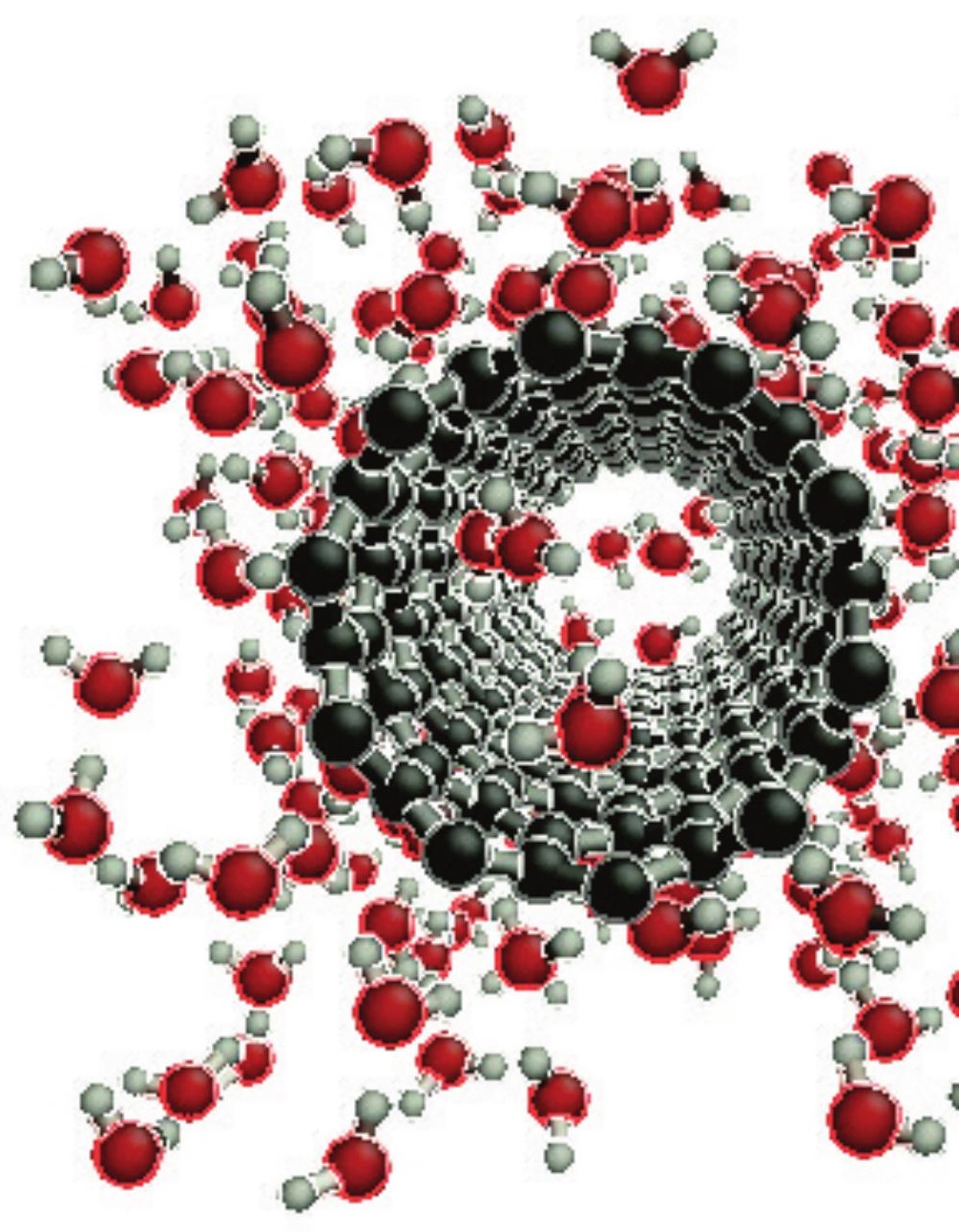

